\title{
Robust Calculation of Chromatic Dispersion Coefficients of Optical Fibers From Numerically Determined Effective Indices Using Chebyshev-Lagrange Interpolation Polynomials
}

\author{
Po-Jui Chiang, Chin-Ping Yu, and Hung-Chun Chang, Senior Member, IEEE, Member, OSA
}

\begin{abstract}
Numerical calculation of chromatic dispersion coefficients of optical fibers is conducted using a procedure involving Chebyshev-Lagrange interpolation polynomials. Only numerically determined effective indices at several wavelengths are needed for obtaining the dispersion curve, and no direct numerical differentiation of the effective refractive index is involved. A silica-filled metallic rectangular waveguide having analytical solutions for the effective refractive index and the chromatic dispersion is used as an example for confirming the accuracy and efficiency of the proposed method. The method is then also applied to the analysis of holey fibers.
\end{abstract}

Index Terms-Dispersion, fiber properties, optical waveguides, photonic-crystal fibers (PCFs), spectral method.

\section{INTRODUCTION}

$\mathbf{T}$ HE CHROMATIC dispersion coefficient of an optical fiber in units of picoseconds per nanometer kilometer is a key quantity in various analysis and design issues in fiber transmission systems, including recent intensive investigation of photonic-crystal fibers (PCFs) or holey fibers [1]-[3]. It is defined as $D=-(\lambda / c) d^{2} n_{\text {eff }} / d \lambda^{2}$, where $n_{\text {eff }}$ is the effective refractive index of the optical fiber mode, $\lambda$ is the wavelength, and $c$ is the speed of light in a vacuum. For a given fiber or waveguide structure, $n_{\text {eff }}$ can be solved at different wavelengths using various numerical methods. Then, in principle, one simple way to obtain $D$ at a particular wavelength is to perform direct numerical differentiation using the central difference scheme based on three $n_{\text {eff }}$ values at three nearby wavelengths. Therefore, to obtain $D$ values at $N$ wavelengths, one needs to calculate $n_{\text {eff }}$ at $3 N$ wavelengths. From the basic theory of

Manuscript received May 27, 2006; revised August 14, 2006. This work was supported in part by the National Science Council of the Republic of China under Grant NSC94-2215-E-002-022.

P.-J. Chiang and C.-P. Yu are with the Graduate Institute of Electro-Optical Engineering, National Taiwan University, Taipei 106-17, Taiwan, R.O.C. (e-mail: d90941006@ntu.edu.tw; chinpingyu@ntu.edu.tw).

H.-C. Chang is with the Department of Electrical Engineering, Graduate Institute of Electro-Optical Engineering, and the Graduate Institute of Communication Engineering, National Taiwan University, Taipei 106-17, Taiwan, R.O.C. (e-mail: hcchang@cc.ee.ntu.edu.tw).

Color versions of Figs. 1, 3, 4, and 6 are available online at http://ieeexplore.ieee.org.

Digital Object Identifier 10.1109/JLT.2006.883646 the finite difference method, the separation between adjacent wavelengths should be small enough to maintain the required accuracy but should not be too small such that the error would explode [4]. Thus, some suitable $\Delta \lambda$ has to be determined. Furthermore, one might worry about the effect of the numerical accuracy of the calculated $n_{\text {eff }}$ on the degree of accuracy of its second derivative. Kuhlmey et al. [5] have reported in their analysis of holey fibers that they generally considered $\sim 1000$ points per unit $\lambda / \Lambda$, with $\Lambda$ being the pitch of the airhole lattice of the holey fiber, to obtain satisfactory results of dispersion curves.

To ensure accurate determination of $D$, over the past two decades, some more elaborate approaches have been proposed including, for example, that based on the matrix perturbation method [6] and those through formulating two associated problems for evaluating the first and second derivatives of the propagation constant [7]-[10]. In this paper, we propose a simple approach utilizing Chebyshev-Lagrange interpolation polynomials as in the Chebyshev spectral method or the Chebyshev collocation method (CCM) [11] and demonstrate its efficiency and robustness in calculating $D$. For instance, over the wavelength range from 0.6 to $1.5 \mu \mathrm{m}$, calculation of $n_{\text {eff }}$ at only 13 wavelength points would guarantee accurate prediction of the $D$ curves. Most importantly, no numerical differentiations of $n_{\text {eff }}$ are required, and thus, computational burden can be greatly reduced.

The rest of this paper is organized as follows: The CCM is described in Section II. The proposed procedure for chromatic dispersion coefficient calculation is given in Section III, along with the analysis of a silica-filled metallic rectangular waveguide. The application to holey fibers and comparison with published results are presented in Section IV. Conclusions are drawn in Section V.

\section{II. $\mathrm{CCM}$}

We first explain the approximation of a function $f(x)$ in the domain $-1 \leq x \leq 1$ under the CCM [11]. The Chebyshev polynomial $T_{N}(x)$ of degree $N$ is defined as $T_{N}(x)=$ $\cos \left(N \cos ^{-1} x\right)$, where $|x| \leq 1$, and the collocation points to be used are given by the Chebyshev-Gauss-Lobatto points defined as the roots of the polynomial $\left(1-x^{2}\right) T_{N}^{\prime}$, where 


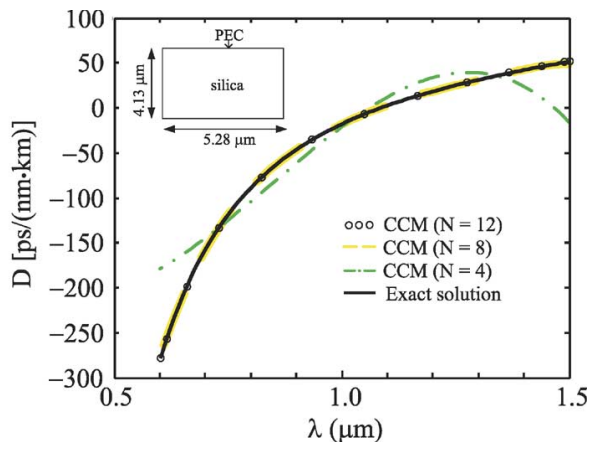

Fig. 1. Chromatic dispersion coefficient curve for the $\mathrm{TE}_{10}$ mode of a silicafilled metallic rectangular waveguide obtained from the exact solution and by the CCM of different degrees.

the prime denotes the derivative. One merit to employ the Chebyshev polynomial is the available analytical formula for its collocation points, which is given by $x_{i}=\cos (i \pi / N)$, $i=0,1,2, \ldots, N$. The CCM provides an $N$ th-order approximation of $f(x)$ as $\left(L_{N} f\right)(x)=\sum_{i=0}^{N} f\left(x_{i}\right) g_{i}(x)$, where the Chebyshev-Lagrange interpolation polynomials are given by $g_{i}(x)=\left[\left(1-x^{2}\right) T_{N}^{\prime}(x)(-1)^{i+1}\right] /\left[c_{i} N^{2}\left(x-x_{i}\right)\right]$ with $c_{0}=$ $c_{N}=2$, and $c_{i}=1$ for $1 \leq i \leq N-1$. The polynomials $g_{i}(x)$ have the properties that $g_{i}\left(x_{j}\right)=1$ for $i=j$ and $g_{i}\left(x_{j}\right)=0$ for $i \neq j$. Therefore, $\left(L_{N} f\right)\left(x_{i}\right)=f\left(x_{i}\right)$ and the derivatives of $f(x)$ at a collocation point $x_{i}$ can be computed by a matrix operator, with the matrix entries $D_{i j}=g_{j}^{\prime}\left(x_{i}\right)$, through $d f\left(x_{i}\right) / d x=\sum_{j=0}^{N} g_{j}^{\prime}\left(x_{i}\right) f\left(x_{j}\right)=\sum_{j=0}^{N} D_{i j} f\left(x_{j}\right)$. The explicit expressions for $D_{i j}$, as given in [11], are $D_{i j}=$ $\left(c_{i} / c_{j}\right)\left[(-1)^{i+j} /\left(x_{i}-x_{j}\right)\right]$ for $i \neq j, i, j=0,1,2, \ldots, N$, $D_{i i}=-x_{i} /\left[2\left(1-x_{i}^{2}\right)\right]$ for $1 \leq i \leq N-1, D_{00}=\left(2 N^{2}+1\right) / 6$, and $D_{N N}=-\left(2 N^{2}+1\right) / 6$. The matrix with elements $D_{i j}$ is termed the differential matrix and will be denoted as $\overline{\overline{\mathbf{D}}}$ in the following. In summary, in the CCM, the function $f(x)$ is approximated by a summation of $N+1$ terms involving $N+1$ known continuous interpolating functions $g_{i}(x)$, and the numerical derivative of $f(x)$ is easily evaluated in terms of the known derivative values of $g_{i}(x)$.

\section{IDEALISTIC WAVEGUIDE HAVING ANALYTICAL CHROMATIC DISPERSION CHARACTERISTICS}

We describe in this section our proposed method and examine its accuracy in calculating chromatic dispersion coefficient curves by referring to some analytical results of an idealistic waveguide, as shown in the inset of Fig. 1. It is a silica-material-filled metallic rectangular waveguide with side widths $a=5.28 \mu \mathrm{m}$ and $b=4.13 \mu \mathrm{m}$. The waveguide is assumed to be enclosed by perfect electric conductor (PEC) walls. From the theory of metallic waveguide [12], the propagation constant of the fundamental $\mathrm{TE}_{10}$ mode has the expression $\beta=\left[k_{0}^{2} \varepsilon-(\pi / a)^{2}\right]^{1 / 2}=n_{\text {eff }} k_{0}$, or we have $n_{\text {eff }}=$ $\left[\varepsilon-(\lambda / 2 a)^{2}\right]^{1 / 2}$, where $k_{0}$ is the wavenumber in free space, and $\varepsilon$ is the relative permittivity of silica. Even when taking into account the material dispersion of silica described by the four-term Sellmeier formulas [13], [14] for $\varepsilon$ as a function of $\lambda$, the derivatives of $n_{\text {eff }}$ with respect to $\lambda$ can be analytically
TABLE I

SELlMeIER COEFFICIENTS [13], [14]

\begin{tabular}{ccccc}
\hline$i$ & 1 & 2 & 3 & 4 \\
\hline$A_{i}$ & 0.53186932 & 0.57234217 & 0.066425330 & 1.1524322 \\
$B_{i}$ & 0.056857781 & 0.11075886 & 6.6776876 & 12.206844 \\
\hline
\end{tabular}

derived, and thus, $D$ versus $\lambda$ has an analytical expression. The related formulas are given as follows:

$$
\begin{aligned}
\frac{d n_{\mathrm{eff}}}{d \lambda}= & \left(\frac{1}{2} \frac{d \varepsilon}{d \lambda}-\frac{\lambda}{4 a^{2}}\right)\left[\varepsilon-\left(\frac{\lambda}{2 a}\right)^{2}\right]^{-1 / 2} \\
\frac{d^{2} n_{\mathrm{eff}}}{d \lambda^{2}}= & \left(\frac{1}{2} \frac{d^{2} \varepsilon}{d \lambda^{2}}-\frac{1}{4 a^{2}}\right)\left[\varepsilon-\left(\frac{\lambda}{2 a}\right)^{2}\right] \\
& -\left(\frac{1}{2} \frac{d \varepsilon}{d \lambda}-\frac{\lambda}{4 a^{2}}\right)^{2}\left[\varepsilon-\left(\frac{\lambda}{2 a}\right)^{2}\right]^{-3 / 2} \\
\varepsilon= & +\sum_{i=1}^{4} A_{i} \frac{\lambda^{2}}{\lambda^{2}-B_{i}^{2}} \\
\frac{d \varepsilon}{d \lambda}= & \sum_{i=1}^{4} A_{i}\left[2 \lambda\left(\lambda^{2}-B_{i}^{2}\right)^{-1}-2 \lambda^{3}\left(\lambda^{2}-B_{i}^{2}\right)^{-2}\right] \\
= & \sum_{i=1}^{4} A_{i} \frac{-2 \lambda B_{i}^{2}}{\left(\lambda^{2}-B_{i}^{2}\right)^{2}} \\
\frac{d^{2} \varepsilon}{d \lambda^{2}}= & \sum_{i=1}^{4} A_{i}\left[2\left(\lambda^{2}-B_{i}^{2}\right)^{-1}-10 \lambda^{2}\left(\lambda^{2}-B_{i}^{2}\right)^{-2}\right. \\
= & \sum_{i=1}^{4} A_{i} \frac{6 \lambda^{2} B_{i}^{2}+2 B_{i}^{4}}{\left(\lambda^{2}-B_{i}^{2}\right)^{3}}
\end{aligned}
$$

with the Sellmeier coefficients [13], [14] given in Table I. The exact analytical result is shown in Fig. 1 as the solid line for wavelength varying from 0.6 to $1.5 \mu \mathrm{m}$.

To apply the CCM, we first do a linear coordinate transformation by mapping the domain $\lambda_{1} \leq \lambda \leq \lambda_{2}$ to the domain $-1 \leq$ $x \leq 1$ of the $x$-axis. By employing Chebyshev polynomial of degree $N=12$, we have 13 Chebyshev-Gauss-Lobatto points in the domain $-1 \leq x \leq 1$ and the corresponding ones in the wavelength domain $\lambda_{1} \leq \lambda \leq \lambda_{2}$. We calculate $n_{\text {eff }}$ of the fundamental core mode of the rectangular waveguide of Fig. 1 at these 13 wavelengths and use these 13 numbers to construct a $13 \times 1$ column vector $\left[n_{\text {eff }}\right]$. The mode solver we use is that based on the finite-difference frequency-domain (FDFD) method [15]. Then, the $D$ values to be determined at these 13 wavelengths are taken to be the entries of the $13 \times 1$ vector $[D]$. It is easy to obtain the following relationship using the simple chain rule given as follows:

$$
[D]=-\left(4 \lambda / c\left(\lambda_{2}-\lambda_{1}\right)^{2}\right) \overline{\overline{\mathbf{D}}} \overline{\overline{\mathbf{D}}}\left[n_{\mathrm{eff}}\right] .
$$

Therefore, once the vector $\left[n_{\text {eff }}\right]$ is known, the vector $[D]$ can be obtained readily, and no numerical differentiation is needed. The 13 circles in Fig. 1 show such results, and it is seen that they agree with the analytical solution. In fact, 
TABLE II

COMPARISON OF FDFD CALCULATIONS OF THE EFFECTIVE INDICES OF THE TE 10 Mode of the Silica-Filled Metallic Rectangular WAVEGUIDE USING DIFFERENT GRID SIZES

\begin{tabular}{ccccc}
\hline & & \multicolumn{3}{c}{$n_{\text {eff }(\mathrm{FDFD})}$} \\
\cline { 3 - 5 }$\lambda(\mu \mathrm{m})$ & $n_{\text {eff (exact) }}$ & $\Delta=0.02 \mu \mathrm{m}$ & $\Delta=0.1 \mu \mathrm{m}$ & $\Delta=0.66 \mu \mathrm{m}$ \\
\hline 0.6 & 1.456935984 & 1.456935997 & 1.456936321 & 1.456950149 \\
0.615333378 & 1.456384568 & 1.456384582 & 1.456384922 & 1.456399471 \\
0.660288568 & 1.454925468 & 1.454925484 & 1.454925876 & 1.454942646 \\
0.731801948 & 1.452960106 & 1.452960126 & 1.452960609 & 1.452981235 \\
0.825 & 1.450804504 & 1.450804530 & 1.450805144 & 1.450831397 \\
0.933531430 & 1.448600938 & 1.448600971 & 1.448601758 & 1.448635425 \\
1.05 & 1.446393699 & 1.446393741 & 1.446394739 & 1.446437394 \\
1.166468570 & 1.444220438 & 1.444220490 & 1.444221723 & 1.444274446 \\
1.275 & 1.442158385 & 1.442158446 & 1.442159922 & 1.442223002 \\
1.368198052 & 1.440328883 & 1.440328954 & 1.440330655 & 1.440403386 \\
1.439711432 & 1.438876976 & 1.438877055 & 1.438878940 & 1.438959554 \\
1.484666622 & 1.437939976 & 1.437940060 & 1.437942066 & 1.438027848 \\
1.5 & 1.437615822 & 1.437615907 & 1.437617956 & 1.437705539 \\
\hline & & & &
\end{tabular}

TABLE III

COMPARISON OF CHROMATIC DISPERSION COEFFICIENTS OBTAINED BASED ON THE CCM USING THE CORRESPONDING $n_{\text {eff(FDFD) }}$ VALUES OF TABLE II

\begin{tabular}{ccccc}
\hline & & \multicolumn{3}{c}{$D_{(\mathrm{FDFD})}$} \\
\cline { 3 - 5 }$\lambda(\mu \mathrm{m})$ & $D_{\text {(exact) }}$ & $\Delta=0.02 \mu \mathrm{m}$ & $\Delta=0.1 \mu \mathrm{m}$ & $\Delta=0.66 \mu \mathrm{m}$ \\
\hline 0.6 & -279.57546 & -279.71641 & -279.73093 & -279.89621 \\
0.615333378 & -255.40079 & -255.57194 & -255.58123 & -255.74523 \\
0.660288568 & -197.17813 & -197.31545 & -197.31970 & -197.48975 \\
0.731801948 & -131.92866 & -132.02038 & -132.02422 & -132.21570 \\
0.825 & -76.980051 & -77.033294 & -77.038416 & -77.255058 \\
0.933531430 & -36.355595 & -36.381035 & -36.387245 & -36.631981 \\
1.05 & -7.6173125 & -7.6229400 & -7.6288289 & -7.9081494 \\
1.166468570 & 12.7538208 & 12.7627199 & 12.7546607 & 12.4442826 \\
1.275 & 27.4139305 & 27.4319495 & 27.4250396 & 27.0821414 \\
1.368198052 & 37.9524554 & 37.9783835 & 37.9690882 & 37.5987286 \\
1.439711432 & 45.1796912 & 45.2109879 & 45.2021777 & 44.8129293 \\
1.484666622 & 49.4420704 & 49.4835971 & 49.4604531 & 49.0465975 \\
1.5 & 50.8551331 & 50.8765939 & 50.8333938 & 50.4053730 \\
\hline
\end{tabular}

we can obtain a continuous curve for $D$ since $d^{2} f(x) / d x^{2}=$ $\sum_{j=0}^{N}\left(d^{2} g_{j}(x) / d x^{2}\right) f\left(x_{j}\right)$. In addition, if we plot the obtained continuous curve for $D$ in Fig. 1, it would be indistinguishable from the solid line. To examine what the enough value for $N$ would be, we have considered $N=8$ and 4 , and the two continuous curves are plotted in Fig. 1. For this waveguide, we found that the $N=8$ curve is almost consistent with the $N=12$ one, but the $N=4$ curve is far from enough. We have found that $N=12$ would provide a very good result for waveguide structures generally encountered, including the examples to be discussed in the next section.

In the FDFD method [15], the waveguide cross section is made up of uniform finite-difference grids (Yee mesh). It is well known that numerical accuracy in the calculation of $n_{\text {eff }}$ would increase with decreasing grid size. Assuming equal numerical grid sizes in the horizontal and vertical directions $(\Delta x=$ $\Delta y=\Delta$ ), we have examined the possible dependence of the accuracy of $D$ on the numerical accuracy of the calculated $n_{\text {eff. }}$. Table II lists the calculated $n_{\text {eff }}$ values for $\Delta=$ $0.02,0.1$, and $0.66 \mu \mathrm{m}$, respectively, along with the exact values at the 13 collocation wavelength points, and Table III lists the corresponding $D$ values obtained using the CCM with $N=13$ along with the exact ones. It is observed in Table II that for $\Delta=0.02 \mu \mathrm{m}$, the FDFD-calculated $n_{\text {eff }}$ has seven

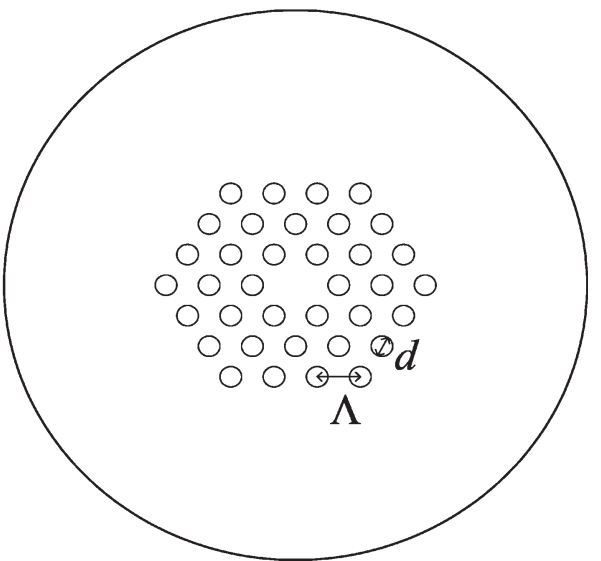

(a)

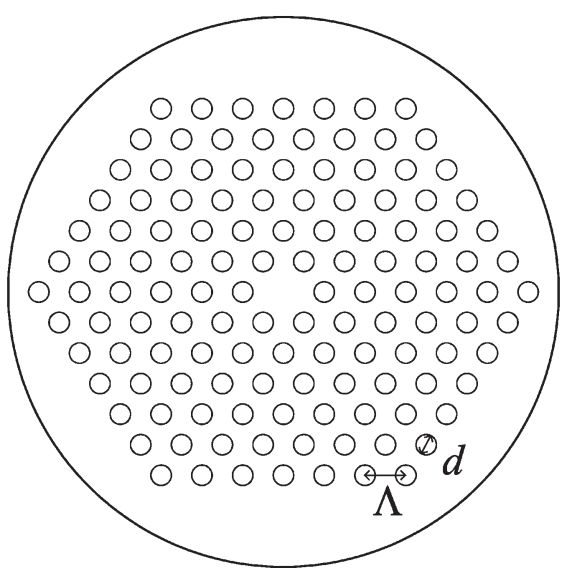

(b)

Fig. 2. (a) Cross section of the three-ring holey fiber. (b) Cross section of the six-ring holey.

digits of accuracy after the decimal point at shorter wavelengths and six digits of accuracy at longer wavelengths and that for $\Delta=0.66 \mu \mathrm{m}$, it decreases to four digits and three digits, respectively. It is found in Table III that with $\Delta=0.02 \mu \mathrm{m}$, the error in the prediction of $D$ is less than $0.09 \%$ over the wavelength range considered and that even with quite coarse grids of $\Delta=0.66 \mu \mathrm{m}$, the error is at most $3.82 \%$ near the zerodispersion wavelength. The proposed method for calculating $D$ curves is, thus, quite robust as far as good numerical accuracy is concerned. That is, with only three to four digits of accuracy after the decimal point for $n_{\text {eff }}, D$ curves of reasonable accuracy can be obtained for this case. Such accuracy in $n_{\text {eff }}$ can be easily achieved using typical waveguide eigenmode solvers based on different numerical methods.

\section{DisPERSION IN Holey FibERS}

One of the attractive features of holey fibers is the better flexibility in designing the $D$ curves through varying the geometrical arrangement of the air holes. Here, we first compare our calculation with that of Kuhlmey et al. [5] by considering the lowest core mode of one three-air-hole-ring structure in their Fig. 3. As mentioned in Section I, Kuhlmey et al. [5] generally considered $\sim 1000$ points per unit $\lambda / \Lambda$ in their calculation to obtain satisfactory results of the $D$ curves. The 


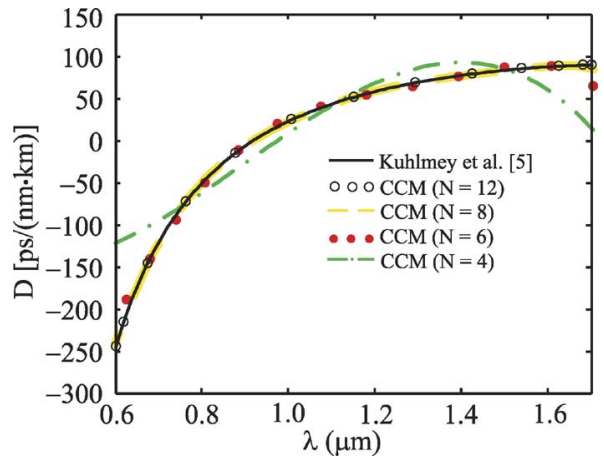

Fig. 3. Chromatic dispersion coefficient curve for the lowest core mode of the three-ring holey fiber of Fig. 2(a), with $\Lambda=2.3 \mu \mathrm{m}$ and $d / \Lambda=0.6$ obtained by the CCM of different degrees. Also shown is the result calculated by Kuhlmey et al. [5].

cross section of the three-ring holey fiber is shown in Fig. 2(a). The parameters considered are $\Lambda=2.3 \mu \mathrm{m}$ and $d / \Lambda=0.6$, where $d$ is the diameter of each air hole. Again, we employ our Yee-mesh-based FDFD waveguide eigenmode solver [15] using the grid size $\Delta x=\Delta y=\Delta=0.1 \mu \mathrm{m}$ to calculate $n_{\mathrm{eff}}$ at 13 wavelengths from $\lambda=0.6$ to $1.7 \mu \mathrm{m}$, corresponding to the Chebyshev-Gauss-Lobatto points for $N=12$. The material dispersion of silica has been taken into account by using the four-term Sellmeier formulas [13], [14]. Then, the $D$ curve is determined using the CCM as in Section III. The $D$ values at the 13 collocation points are presented as the circles in Fig. 3 with the solid line being that shown in [5, Fig. 3]. The agreement is obviously very good. If we plot our calculated continuous curve for $D$ in Fig. 3, it would, again, be indistinguishable from the solid line. We have also considered $N=8,6$, and 4 , and the three continuous curves are plotted in Fig. 3. Again, $N=4$ is not enough for obtaining correct curve. As $N$ is increased, the results converge with the maximum errors occurring near the two ends. This is the characteristic of the Chebyshev spectral method. It can be seen that there exist some noticeable differences at the two ends between the $N=8$ and $N=12$ lines. This example demonstrates a general feature that a suitable value of degree $N$ can be determined by examining the numerical convergence at the ends of the wavelength domain. As long as the difference between the results for degrees $N-1$ and $N$, respectively, at the ends is small enough for specific application, the whole curve of degree $N$ will surely be acceptable.

In our FDFD eigenmode solver, as discussed in [15], we have two schemes in treating the dielectric interface problem, that is, the interface between silica and air, namely 1) the simple staircase approximation and 2) the more elaborative boundary condition (BC) matching method. The latter has been shown to be much more accurate than the former. We have analyzed the structure of Fig. 3 using both schemes, and the results are given in Table IV. It can be observed that although the $n_{\text {eff }}$ values of the staircase scheme agree with those of the $\mathrm{BC}$ matching scheme only up to three digits after the decimal point, the difference in $D$ results is at most $0.64 \%$, which occurs near $\lambda=1.7 \mu \mathrm{m}$. Again, this demonstrates the robustness of the proposed method for calculating $D$.

We then consider a six-ring holey fiber recently analyzed by Saitoh and Koshiba using the finite element method
TABLE IV

COMPARISON OF EFFECTIVE INDICES AND CHROMATIC DISPERSION COEFFICIENTS OBTAINED BASED ON THE FDFD METHOD USING THE BOUndary Matching SCHEME AND the STAIRCASE APPROXIMATION, RESPECTIVELY, FOR THE CASE OF Fig. 3

\begin{tabular}{ccccc}
\hline & \multicolumn{2}{c}{$n_{\text {eff }}$} & \multicolumn{2}{c}{$D(\mathrm{ps} /(\mathrm{nm} \cdot \mathrm{km}))$} \\
\cline { 2 - 5 }$\lambda(\mu \mathrm{m})$ & BC matching & Stair-case & BC matching & Stair-case \\
\hline 0.60000000 & 1.452803772 & 1.452869660 & -244.20102 & -244.28426 \\
0.61874080 & 1.451886368 & 1.451955124 & -214.48060 & -214.55726 \\
0.67368603 & 1.449387996 & 1.449465231 & -145.20513 & -145.26017 \\
0.76109127 & 1.445789815 & 1.445880675 & -72.028534 & -72.042572 \\
0.87500000 & 1.441415901 & 1.441524562 & -14.212620 & -14.162906 \\
1.00764953 & 1.436405850 & 1.436534948 & 26.0077923 & 26.142342 \\
1.15000000 & 1.430885430 & 1.431035672 & 52.6105505 & 52.843172 \\
1.29235047 & 1.425092844 & 1.425263008 & 69.7143729 & 70.046610 \\
1.42500000 & 1.419403070 & 1.419590405 & 80.2509002 & 80.672640 \\
1.53890873 & 1.414281095 & 1.414481939 & 86.2323821 & 86.725200 \\
1.62631397 & 1.410203107 & 1.410413477 & 89.2158539 & 89.757865 \\
1.68125920 & 1.407575091 & 1.407791061 & 90.4180097 & 90.987959 \\
1.70000000 & 1.406667539 & 1.406885349 & 90.6087483 & 91.187627 \\
\hline
\end{tabular}

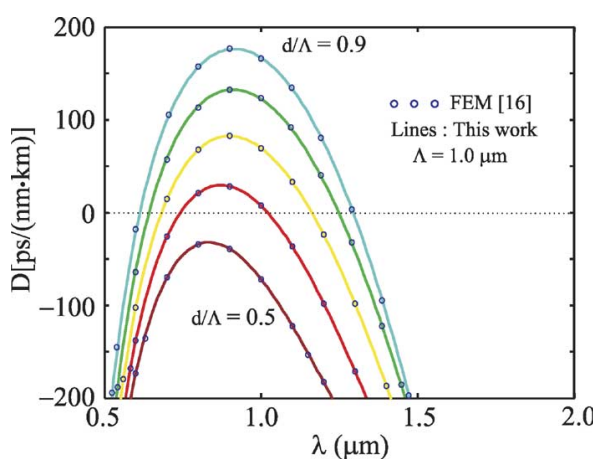

Fig. 4. Chromatic dispersion coefficient curves for the lowest core mode of the six-ring holey fiber of Fig. 2(b), with $\Lambda=1.0 \mu \mathrm{m}$ for different $d / \Lambda$ values obtained by the CCM of different degrees. Also shown are sample points extracted from the results calculated by Saitoh and Koshiba [16, Fig. 16(a)].

(FEM) [16], with the cross section as shown in Fig. 2(b). In [16, Fig. 16(a)], six $D$ curves of the lowest core mode were plotted for $d / \Lambda=0.5,0.6,0.7,0.8$, and 0.9 , respectively, and $\Lambda=1.0 \mu \mathrm{m}$. We employ the FDFD eigenmode solver [15] using the grid size $\Delta x=\Delta y=\Delta=0.067 \mu \mathrm{m}$ and the CCM with $N=12$ to obtain the corresponding results and plot them as the five lines in Fig. 4. These five lines agree well with those in [16, Fig. 16(a)]. The circles are sample points extracted from the results of [16].

In the numerical examples presented so far, as in most situations in the holey-fiber study, the dispersion variations were seen to vary by over hundreds of picoseconds per nanometer kilometer within the interested wavelength range. We finally consider an extreme situation in which the holey fiber is designed to possess nearly zero dispersion-flattened characteristics. One such structure was demonstrated in [16], with the cross section as shown in Fig. 5 and the dispersion designed to remain between 0.1 and $0.3 \mathrm{ps} /(\mathrm{nm} \cdot \mathrm{km})$ for the wavelength range from 1.41 to $1.68 \mu \mathrm{m}$. The structure parameters of this ten-air-hole-ring holey fiber were $\Lambda=1.6 \mu \mathrm{m}, d_{1}=0.47 \mu \mathrm{m}$, $d_{2}=0.71 \mu \mathrm{m}, d_{3}=0.74 \mu \mathrm{m}, d_{4}=0.62 \mu \mathrm{m}$, and $d_{5}=\cdots=$ $d_{10}=0.65 \mu \mathrm{m}$, where $d_{i}$ is the air-hole diameter of the $i$ th air-hole ring. For this structure, we have found that the numerical accuracy in the calculation of $n_{\text {eff }}$ is quite critical in determining the $D$ curve. As shown in Fig. 6, the dots are 


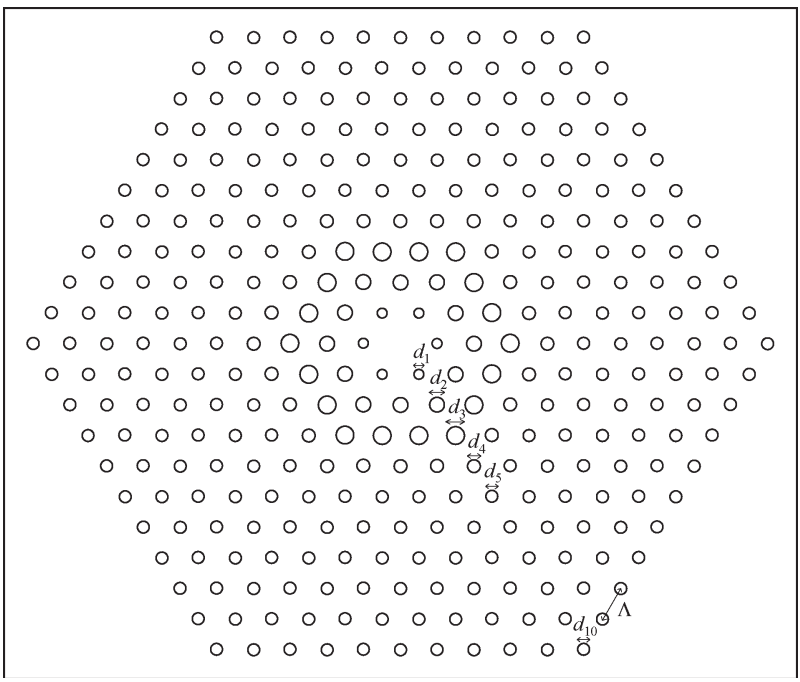

Fig. 5. Cross section of the nearly zero dispersion-flattened holey fiber with ten air-hole rings.

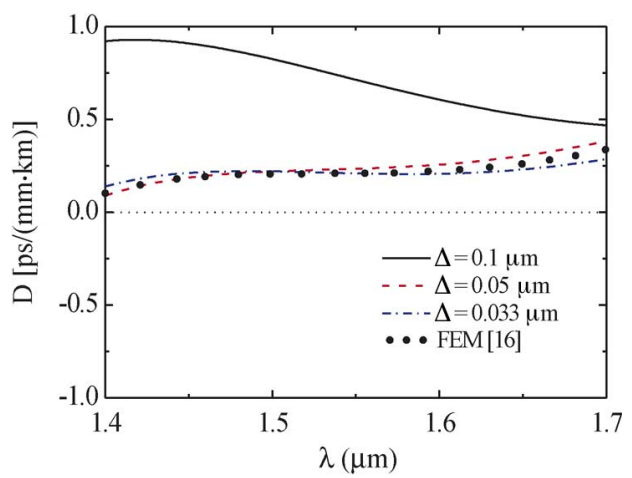

Fig. 6. Chromatic dispersion coefficient curve for the lowest core mode of the ten-ring holey fiber of Fig. 5, with $\Lambda=1.6 \mu \mathrm{m}$ and a designed air-hole diameter distribution obtained by the FDFD eigenmode solver using different grid sizes and the CCM of degree 12. Also shown are sample points extracted from the results calculated by Saitoh and Koshiba [16, Fig. 19].

sample points extracted from the $D$ curve of the lowest core mode given in [16, Fig. 19], and the three continuous lines are obtained using our FDFD eigenmode solver with the grid sizes $\Delta x=\Delta y=\Delta=0.1,0.05$, and $0.033 \mu \mathrm{m}$, respectively, and the CCM with $N=12$. The BC matching scheme has been employed in the FDFD calculation of $n_{\text {eff }}$ to ensure high accuracy. It is seen that the dispersion calculation for this case is quite sensitive to the grid size in the FDFD method. The grid size of $0.1 \mu \mathrm{m}$ is obviously not fine enough for this calculation. It should be emphasized here that the CCM still works as an efficient scheme in determining the $D$ curve from the $n_{\text {eff }}$ calculation. The key point is that to obtain enough accurate $D$ curve, we need to have $n_{\text {eff }}$ values of enough high accuracy, and in the present case, the demand of the $n_{\text {eff }}$ accuracy is quite high, as can be understood from the following discussion.

We approximate the $D$ curve to be determined for the present holey fiber in the wavelength range of Fig. 6 by the expression $A+B \sin [\pi(\bar{\lambda}-1.55) / 0.3]$, which can be considered as the first two terms in a Taylor series expansion, where $\bar{\lambda}=\lambda$ / $(1 \mu \mathrm{m})$ is a dimensionless variable. According to the result of [16] in Fig. 6, $A \approx 0.2 \mathrm{ps} /(\mathrm{nm} \cdot \mathrm{km})$ and $B \approx 0.1 \mathrm{ps} /(\mathrm{nm} \cdot \mathrm{km})$.
The expression above should be equal to $-(\lambda / c) d^{2} n_{\text {eff }} / d \lambda^{2}$, which can be approximately written as $-\left[\left(\lambda_{0} / c\right) /(1 \mu \mathrm{m})^{2}\right]$ $d^{2} n_{\mathrm{eff}} / d \bar{\lambda}^{2}=-(1.55 / 3) \times 10^{4} d^{2} n_{\mathrm{eff}} / d \bar{\lambda}^{2} \mathrm{ps} /(\mathrm{nm} \cdot \mathrm{km})$, with $\lambda_{0}$ being taken to be $1.55 \mu \mathrm{m}$. Thus, we have $0.2+0.1 \sin [\pi(\bar{\lambda}-$ $1.55) / 0.3] \approx-(1.55 / 3) \times 10^{4} d^{2} n_{\mathrm{eff}} / d \bar{\lambda}^{2}$, which gives $n_{\mathrm{eff}} \approx$ $-(0.6 / 1.55) \times 10^{-4}\left(C_{2}+C_{1} \bar{\lambda}+\bar{\lambda}^{2} / 2\right)-(0.3 / 1.55)(0.3 / \pi)^{2} \times$ $10^{-4} \sin [\pi(\bar{\lambda}-1.55) / 0.3]=-3.87 \times 10^{-5}\left(C_{2}+C_{1} \bar{\lambda}+\bar{\lambda}^{2} / 2\right)-$ $1.76 \times 10^{-7} \sin [\pi(\bar{\lambda}-1.55) / 0.3]$, where $C_{1}$ and $C_{2}$ are constants. It can be seen that due to the small value of $B(\approx 0.1 \mathrm{ps} /(\mathrm{nm} \cdot \mathrm{km}))$, the amplitude of the sine term in $n_{\text {eff }}$ is as small as in the order of $10^{-7}$, which implies that one needs to achieve very high accuracy in the numerical calculation of $n_{\text {eff }}$ in order to be able to resolve this sine variation. This implication is also true even when $A$ is not small (dispersion-flattened but not nearly zero). When $B$ is in the order of hundreds of picoseconds per nanometer kilometer as in the earlier examples, the amplitude of the sine term becomes three orders larger, and the demand of the numerical accuracy of $n_{\text {eff }}$ is greatly reduced.

\section{CONCLUSION}

We have proposed a simple procedure for the numerical calculation of chromatic dispersion coefficients of optical fibers. The procedure employs the CCM involving Chebyshev-Lagrange interpolation polynomials. No direct numerical differentiation of the propagation constant or of the effective index is involved. From the application of the proposed method to an idealistic silica-filled metallic waveguide, which provides analytical solutions for the effective indices and chromatic dispersion coefficients of the waveguide modes, we have demonstrated the robustness of this simple scheme and its efficiency and accuracy. Our analysis of a three- and a sixring holey fiber also shows very good agreement with published results based on different methods. We have found that calculating the effective indices at 13 wavelengths, corresponding to the Chebyshev-Gauss-Lobatto collocation points for $N=12$, would generally provide very good results for the $D$ curves. The proposed method can be very easily applied to any structure by using (6) with the entries of the differential matrix $\overline{\mathbf{D}}$ given at the end of Section II. It should be noticed that in the determination of a dispersion-flattened curve, as demonstrated in the final example of the ten-ring holey fiber, the CCM still works well as an efficient scheme, but the achievement of the correct result relies highly on the numerical accuracy of the effective index calculation.

\section{ACKNOWLEDGMENT}

The authors would like to thank the National Center for High-Performance Computing, Hsinchu, Taiwan, R.O.C., for providing useful computing resources.

\section{REFERENCES}

[1] D. Mogilevtsev, T. A. Birks, and P. S. J. Russell, "Group-velocity dispersion in photonic crystal fibers," Opt. Lett., vol. 23, no. 21, pp. 1662-1664, Nov. 1998.

[2] T. A. Birks, D. Mogilevtsev, J. C. Knight, and P. S. J. Russell, "Dispersion compensation using single-material fibers," IEEE Photon. Technol. Lett., vol. 11, no. 6, pp. 674-676, Jun. 1999. 
[3] J. C. Knight, J. Arriaga, T. A. Birks, A. Ortigosa-Blanch, W. J. Wadsworth, and S. J. Russell, "Anomalous dispersion in photonic crystal fiber," IEEE Photon. Technol. Lett., vol. 12, no. 7, pp. 817-819, Jul. 2000.

[4] J. H. Mathews and K. D. Fink, Numerical Method Using MATLAB, 3rd ed. Upper Saddle River, NJ: Prentice-Hall, 1999, ch. 6.

[5] B. T. Kuhlmey, T. P. White, G. Renversez, D. Maystre, L. C. Botten, C. M. de Sterke, and R. C. McPhedran, "Multipole method for microstructured optical fibers-II: Implementation and results," J. Opt. Soc. Amer. B, Opt. Phys., vol. 19, no. 10, pp. 2331-2340, Oct. 2002.

[6] A. Sharma and S. Banerjee, "Chromatic dispersion in single mode fibers with arbitrary index profiles: A simple method for exact numerical evaluation,” J. Lightw. Technol., vol. 7, no. 12, pp. 1919-1923, Dec. 1989.

[7] E. K. Sharma, A. Sharma, and I. C. Goyal, "Propagation characteristics of single mode optical fibers with arbitrary index profiles: A simple numerical approach," IEEE J. Quantum Electron., vol. QE-18, no. 10, pp. 1484-1489, Oct. 1982.

[8] H. Y. Lin, R. B. Wu, and H. C. Chang, "An efficient algorithm for determining the dispersion characteristics of single-mode optical fibers," J. Lightw. Technol., vol. 10, no. 6, pp. 705-711, Jun. 1992.

[9] T. L. Wu and H. C. Chang, "An efficient numerical approach for determining the dispersion characteristics of dual-mode elliptical-core optical fibers," J. Lightw. Technol., vol. 13, no. 9, pp. 1926-1934, Sep. 1995.

[10] T. L. Wu, J. S. Chiang, and C. H. Chao, "A novel approach for calculating the dispersions of photonic crystal fibers," IEEE Photon. Technol. Lett., vol. 16, no. 6, pp. 1492-1494, Jun. 2004.

[11] C. Canuto, M. Y. Hussani, A. Quarteroni, and T. Zang, Spectral Methods in Fluid Dynamics. New York: Springer-Verlag, 1988.

[12] D. K. Cheng, Field and Wave Electromagnetics, 2nd ed. Redwood City, CA: Addison-Wesley, 1989, p. 551.

[13] I. H. Maliston, "Interspecimen comparison of the refractive index of fused silica," J. Opt. Soc. Amer., vol. 55, no. 10, pp. 1205-1209, 1965.

[14] B. Brixner, "Refractive-index interpolation for fused silica," J. Opt. Soc. Amer., vol. 57, no. 5, pp. 674-676, May 1967.

[15] C. P. Yu and H. C. Chang, "Yee-mesh-based finite difference eigenmode solver with PML absorbing boundary conditions for optical waveguides and photonic crystal fibers," Opt. Express, vol. 12, no. 25, pp. 6165-6177, Dec. 2004.

[16] K. Saitoh and M. Koshiba, "Numerical modeling of photonic crystal fibers," J. Lightw. Technol., vol. 23, no. 11, pp. 3580-3590, Nov. 2005.

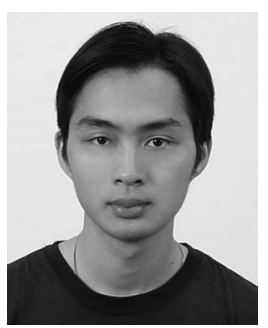

Po-Jui Chiang was born in Kaohsiung, Taiwan, R.O.C., on November 26, 1974. He received the B.S. degree in electronics engineering from the National Taiwan University of Science and Technology, Taipei, Taiwan, in 1998 and the M.S. degree in electro-optical engineering from the National Taiwan University, Taipei, in 2000. He is currently working toward the Ph.D. degree at the Graduate Institute of Electro-Optical Engineering, National Taiwan University. His research interests include numerical methods for solving optical waveguide problems.

$\mathrm{He}$ is also currently with the Graduate Institute of Electro-Optical Engineering, National Taiwan University. His research interests include numerical methods for solving optical waveguide problems.

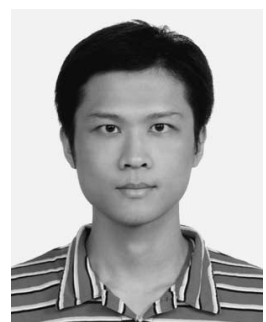

Chin-Ping Yu was born in Tainan, Taiwan, R.O.C., on January 24, 1975. He received the B.S. degree in electrical engineering and the Ph.D. degree in communication engineering from the National Taiwan University, Taipei, Taiwan, in 1997 and 2004, respectively.

Since 2004, he has been a Post-Doctoral Research Fellow with the Graduate Institute of Electro-Optical Engineering, National Taiwan University. His research interests include numerical methods for solving optical waveguide problems and applications of photonic-crystal structures.

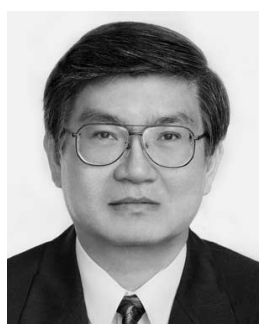

Hung-Chun Chang (S'78-M'83-SM'00) was born in Taipei, Taiwan, R.O.C., on February 8, 1954 $\mathrm{He}$ received the B.S. degree from the National Taiwan University, Taipei, in 1976 and the M.S. and Ph.D. degrees from Stanford University, Stanford, CA, in 1980 and 1983, respectively, all in electrical engineering.

From 1978 to 1984, he was with the Space, Telecommunications, and Radioscience Laboratory, Stanford University. In August 1984, he joined the faculty of the Department of Electrical Engineering, National Taiwan University, where he is currently a Professor. He served as Vice Chairman of the Department of Electrical Engineering from 1989 to 1991 and as Chairman of the newly established Graduate Institute of Electro-Optical Engineering from 1992 to 1998 . He is also with the Graduate Institute of Communication Engineering, National Taiwan University. His current research interests include the theory, design, and application of guided-wave structures and devices for fiber optics, integrated optics, optoelectronics, and microwaveand millimeter-wave circuits.

Dr. Chang is a member of Sigma Xi, the Phi Tau Phi Scholastic Honor Society, the Chinese Institute of Engineers, the Photonics Society of ChineseAmericans, the Optical Society of America, the Electromagnetics Academy, and the China/SRS (Taipei) National Committee (a Standing Committee Member during 1988-1993 and since 2006) of the International Union of Radio Science (URSI). He has been serving as the Institute of Electronics, Information, and Communication Engineers (Japan) Overseas Area Representative in Taipei, since 2002. In 1987, he was among the recipients of the Young Scientists Award at the URSI XXIInd General Assembly. In 1993, he was one of the recipients of the Distinguished Teaching Award sponsored by the R.O.C., Ministry of Education. In 2004, he received the Merit NSC Research Fellow Award sponsored by the R.O.C., National Science Council. 